Clinical Medicine

Poster

Abstract ID: 102

\title{
A prevalence study into use and management of peripheral intravenous catheters
}

Azlina Daud | Hazimah Rahman | Farrah Ilyani Che Jamaluddin

Kulliyyah of Nursing, International Islamic University Malaysia

Introduction: There are over half of patients admitted to the hospital required peripheral intravascular catheter (PIVC) inserted. However, there are no data reported on the use of PIVC in Malaysia. Thus, this study aims to determine the prevalence of PIVCs use and PIVC complications in patients with PIVC. Methods: An observational study was conducted on patients who had PIVC in medical, surgical, obstetric and orthopedic wards in Hospital Tunku Ampuan Afzan, Kuantan, Malaysia. Results: Over 2 months, the total admission for 4 wards was 1466 and out of this total admission, 490 patients were recruited. Reasons for PIVC insertion were for IV fluids (62.4\%) and for medication administration (31.8\%). Most of the PIVC inserted in the general ward $(91.8 \%)$ followed by from an emergency department. The PIVC insertion was done by the doctor (84.5\%). All patients used the same brand of catheter, which is Vasofix Branulle and used transparent polyurethane dressing. There were $28(5.7 \%)$ patients have signs of thrombophlebitis. The Chi-square test showed that there was a significant association between day of PIVC insertion with thrombophlebitis $(p=0.006)$. Conclusions: In conclusion, the study processes resulted in a proportion of $33.4 \%$ of patients with PIVC with the total number of admission to the 4 wards and $5.7 \%$ patients have signs of thrombophlebitis.

KEYWORDS: Peripheral intravascular catheter, complications, thrombophlebitis 